The effects of inadequate thyroid hormone availability to the brain on adult cognitive function are poorly understood. This study assessed the effects of hypothyroidism on cognitive function using a standard neuropsychological battery in 14 patients suffering from untreated hypothyroidism and complaining of subjective cognitive difficulties in comparison with 10 age-matched healthy comparison subjects. Significant differences between groups were limited to verbal memory retrieval as measured by the California Verbal Learning Test (CVLT). On short delay free recall, long delay free recall, and long delay cued recall, significant differences remained between groups despite the limited statistical power of this study. There were no significant results found between groups on attentional or nonverbal tasks. Results suggest that hypothyroid-related memory deficits are not attributable to an attentional deficit but rather to specific retrieval deficits.

(The Journal of Neuropsychiatry and Clinical Neurosciences 2007; 19:132-136)

\section{Verbal Memory Retrieval Deficits Associated With Untreated Hypothyroidism}

\author{
Karen J. Miller, Ph.D. \\ Thomas D. Parsons, M.A. \\ Peter C. Whybrow, M.D. \\ Katja Van Herle, M.D. \\ Natalie Rasgon, M.D., Ph.D. \\ Andre Van Herle, M.D. \\ Dorothy Martinez, M.D. \\ Dan H. Silverman, M.D. \\ Michael Bauer, M.D., Ph.D.
}

$\mathrm{H}$ ypothyroidism is often associated with mood disturbances and cognitive impairment, implying that thyroid hormones are critical for normal brain functioning. ${ }^{1-4}$ In particular, hypothyroidism has been associated with several cognitive deficits, including general intelligence, psychomotor speed, visual-spatial skills and memory. ${ }^{1,2,5-8}$ In contrast, motor skills, language, inhibitory efficiency, set-shifting and sustained auditory attention appear to be less affected by hypothyroidism. ${ }^{2,6-8}$ However, it is difficult to generalize from these studies given the heterogeneity of the samples used, ranging widely in sample size, population (cancer versus biochemical evidence of hypothyroid-

\footnotetext{
Received December 20, 2004; revised April 3, 2006; accepted April 10, 2006. Drs. Miller, Parsons, Rasgon, Whybrow, and Bauer are associated with the Semel Institute for Neuroscience and Human Behavior, Department of Psychiatry and Biobehavioral Sciences, University of California, Los Angeles, California. Drs. Martinez, K van Herle, and A van Herle are affiliated with the Department of Medicine, Division of Endocrinology, University of California, Los Angeles. Dr. Silverman is affiliated with the Department of Molecular and Medical Pharmacology, David Geffen School of Medicine, University of California, Los Angeles. Dr. Rasgon is also affiliated with the Department of Psychiatry and Behavioral Sciences, Stanford School of Medicine, Palo Alto, California. Dr. Bauer is also affiliated with the Department of Psychiatry and Psychotherapy, Charité-Universitätsmedizin Berlin, Campus Charité Mitte, Berlin, Germany. Address correspondence to Dr. Bauer, Department of Psychiatry and Psychotherapy, Charité-Universitätsmedizin Berlin, Campus Charité Mitte (CCM), Schumannstr. 20/21, 10117 Berlin, Germany; michael.bauer@charite.de (e-mail).
}

Copyright (C) 2007 American Psychiatric Publishing, Inc. 
ism), age, severity of hypothyroidism and in the neuropsychological measures administered.

Recent research has focused on specifying the memory deficits associated with hypothyroidism. Studies have found that patients with biochemical evidence of untreated hypothyroidism exhibit a specific memory retrieval deficit for verbally presented material. Jaeschke et al. $^{9}$ reported a significant improvement in verbal memory with levothyroxine treatment for their 18 participants, using a memory composite of contextual and associative memory tasks. Processing speed did not improve with treatment. Burmeister et al. ${ }^{2}$ found that discontinuing thyroid hormone treatment for 13 thyroid cancer patients led to specific verbal memory retrieval deficits as demonstrated with a learning task.

The goal of this study was to test whether patients with biochemical evidence of untreated hypothyroidism would exhibit a specific memory retrieval deficit for verbally presented material, or whether broader executive difficulties (sustained attention, set-shifting, response inhibition, cognitive flexibility) were associated with memory detriments. Our second hypothesis was that individuals with untreated hypothyroidism would have more difficulty retrieving the verbal information, both after short and long delays, despite being given the opportunity to consolidate the information. After completing our analysis of verbal memory, we decided to carry out additional, unhypothesized, exploratory analyses to examine other possible domains affected by untreated hypothyroidism.

\section{METHOD}

Participants and Procedures

We recruited subjects with untreated hypothyroidism from the clinics of the UCLA Division of Endocrinology. Healthy comparison subjects were recruited through flyers and advertisements in the campus newspaper. Fourteen subjects with hypothyroidism and 10 agematched healthy comparison subjects gave written informed consent and participated in a study approved by the UCLA Institutional Review board. All consecutively enrolled subjects (patients and comparison subjects) underwent screening procedures consisting of a complete medical and physical examination, a routine laboratory evaluation (blood count, blood chemistry, urine drug toxicology), a magnetic resonance imaging
(MRI) scan and a comprehensive neuropsychiatric evaluation. The latter comprised a psychiatric interview, a structured interview for DSM-IV diagnosis, the MINI International Neuropsychiatric Interview, Version 5.0.0, ${ }^{10}$ and a standard neuropsychological battery. Depressive symptoms were assessed with the clinician-rated 17-item Hamilton Depression Rating Scale (HAM-D).

The inclusion criteria were: 1) Individuals with hypothyroidism must have stated that they were experiencing subjective cognitive difficulties; 2 ) hypothyroidism must be confirmed by elevated basal TSH levels; 3 ) both genders must be represented and be between the ages of 18 and 65 years; 4) participants must be considered by the investigator to be compliant; and 5) participants must have an educational level and a degree of understanding such that they can understand the study and give informed consent.

Exclusion criteria included the presence of endocrine or autoimmune diseases other than hypothyroidism and myxedema coma. Pregnant or lactating women who had given birth in the past 12 months were excluded (to rule out antithyroid antibodies positive postpartum thyroiditis), as were women of childbearing potential not using contraception. Further criteria for exclusion were psychotic features, a history of bipolar disorder or schizoaffective disorder, and intake of thyroid hormones or corticosteroids in the previous 2 months. Subjects judged clinically to be at serious risk of suicide and subjects who were not facile in English were also excluded.

Comparison subjects were in good general health and underwent the same psychiatric screening procedures described above for hypothyroid subjects. The inclusion criteria were: 1) Being between the ages of 18 and 65 years; 2 ) having no current or past mental disorder (including a depressive episode), according to the MINI, and a negative history of major organic brain disease; 3) no intake of any hormonal agents or psychotropic agents for at least 2 months prior to study; and 4) no history of endocrine, cardiac or other severe medical disease. Subjects with current substance dependency or significant substance abuse were also excluded from both groups.

\section{Neuropsychological Test Battery}

The neuropsychological battery included a diverse collection of instruments. Verbal memory was assessed with the Logical Memory Test from the Wechsler Memory Scale-III (WMS-III) ${ }^{11}$ and the California Verbal Learning Test (CVLT). ${ }^{12}$ Nonverbal memory was as- 
VERBAL MEMORY RETRIEVAL DEFICITS AND HYPOTHYROIDISM

sessed with the Rey-Osterrieth Complex Figure test. ${ }^{13}$ Attention was assessed with the Trail-Making Test, Part A. ${ }^{14}$ The Trail-Making Test, Part B, and the Controlled Oral Word Association (COWA), ${ }^{15}$ evaluated executive functioning. Language skills were tested with the Animal Naming test (category or semantic verbal fluency). ${ }^{16}$

\section{Data Analysis}

Given the similarity in the hypothyroid and the comparison groups in age, gender, and education of participants, no correction for these variables was employed. To assess which patterns of performance were retained for the hypothyroidism group and the comparison group participants, our data analysis included $t$ tests for independent samples (the data were normally distributed) to evaluate the significance of hypothyroidism and cognition. Given our two hypotheses, we completed two sets of comparisons. The first set included three comparisons (Logical Memory I and II, and CVLT Total), and the second set included five comparisons (CVLT List B, CVLT Short Delay Free, CVLT Short Delay Cued, CVLT Long Delay Free, and CVLT Long Delay Cued). We chose not to utilize an adjustment for multiple comparisons because it tends to be too conservative: by controlling the group-wise error rate, each individual test is held to an unreasonably high standard. This increases the probability of a Type II error and makes it likely that legitimately significant results will fail to be detected. ${ }^{17}$ It is important to note, however, that the use of a Bonferroni correction would not affect the first set of comparisons because these were already nonsignificant. Further, a Bonferroni adjustment does not affect our significant findings for CVLT Short Delay Free or CVLT Long Delay Cued. It does, however, affect CVLT Long Delay Free.

\section{RESULTS}

The groups did not differ significantly in gender, age, education and ethnicity (Table 1). TSH levels of both groups are shown in Table 1.

The descriptive statistics and comparisons for all neuropsychological tests are shown in Table 2. Although verbal group effects were found between the hypothyroid and comparison group, they were limited to verbal memory retrieval on the California Verbal Learning Test (CVLT). On Short Delay Cued Recall $(p=0.05)$, Long Delay Free Recall $(\mathrm{p}<0.05)$ and Long Delay Cued Recall $(p=0.01)$, significant differences remained between groups, despite the limited statistical power of this study. Results from additional unhypothesized exploratory analyses to examine other possible domains affected by untreated hypothyroidism revealed no significant differences between the hypothyroid and control group on attentional or nonverbal tasks.

\section{DISCUSSION}

This study confirms our initial hypothesis that hypothyroidism is associated with memory deficits. The hypothyroid participants did not differ from healthy comparison subjects in terms of encoding new verbal information or the delayed recognition of this same information. Rather, the untreated hypothyroid participants demonstrated a specific deficit in memory retrieval. They had more difficulty retrieving the verbal information after a short delay and appeared to sustain this retrieval problem after a longer delay despite being provided the opportunity to consolidate the information (with category cues after the short delay recall). Nor did category cues help their long delay retrieval, which might have further implications for the significance of the retrieval deficit.

Interestingly, the hypothyroid participants, though they evidenced depressive symptoms in their HAM-D scores, did not demonstrate the cognitive difficulties usu-

\begin{tabular}{|c|c|c|}
\hline & $\begin{array}{c}\text { Healthy } \\
\text { Comparison } \\
\text { Subjects } \\
(\mathbf{N}=10)\end{array}$ & $\begin{array}{c}\text { Hypothyroid } \\
\text { Subjects } \\
(\mathrm{N}=14)\end{array}$ \\
\hline Gender (Female/Male) & $8 / 2$ & $11 / 3$ \\
\hline $\operatorname{Age}^{*}[\min / \max ]$ & $\begin{array}{c}41(\mathrm{SD}=12) \\
{[21 / 64]}\end{array}$ & $\begin{array}{c}42(\mathrm{SD}=12.5) \\
{[22 / 65]}\end{array}$ \\
\hline \multicolumn{3}{|l|}{ Education } \\
\hline Postgraduate & 3 & 4 \\
\hline Completed College & 4 & 3 \\
\hline Some College & 3 & 4 \\
\hline High School Graduate & 0 & 2 \\
\hline \multicolumn{3}{|l|}{ Ethnicity } \\
\hline Caucasian (non-Hispanic) & 5 & 12 \\
\hline $\begin{array}{l}\text { Caucasian of South American } \\
\text { descent }\end{array}$ & 1 & 1 \\
\hline Asian & 2 & 0 \\
\hline African American & 2 & 1 \\
\hline $\mathrm{TSH}^{*}(\mathrm{mcIU} / \mathrm{ml})[\min / \max ]$ & $\begin{array}{c}1.4(\mathrm{SD}=0.5) \\
{[1.1 / 2.4]}\end{array}$ & $\begin{array}{c}31.5(\mathrm{SD}=56.2) \\
\quad[5.4 / 221.8]\end{array}$ \\
\hline $\begin{array}{l}\text { Hamilton Depression Rating Scale } \\
\text { (HAM-D-17) }\end{array}$ & $4.2(\mathrm{SD}=4.0)$ & $14.6(\mathrm{SD}=9.6)$ \\
\hline
\end{tabular}


ally associated with major depression-that is, their performance on tasks of sustained attention, set-shifting, and response inhibition or cognitive flexibility were not significantly different from the performance of comparison subjects. Typically, these are the broad executive difficulties seen in depressed patients and are sometimes associated with memory decline in normal aging. ${ }^{18-21}$

These results extend the data acquired by previous researchers who have demonstrated that memory is a specific deficit in hypothyroidism and that the memory deficit appears to be distinct from the cognitive difficulties usually associated with depressive symptomatology. 2,6,9,22-24

There are similarities and differences between the results of this study and the literature. Our study is similar to that of Burmeister et $a .^{2}$ in that we also found that hypothyroidism was associated with a specific memory retrieval deficit but not with other cognitive deficits (visual-spatial, executive). Interestingly, both studies utilized younger participants than those often examined in the literature on memory and hypothyroidism. 6,7,22 Possibly, previous researchers did not find an isolated effect for memory retrieval because of age-related cognitive changes in their populations. Specifically, visual-spatial functioning and psychomotor speed are known to decline with normal aging.

However, our study is unique because, unlike the population examined by Burmeister et al., ${ }^{2}$ our participants were not thyroid cancer patients and, at the time of evaluation, had not yet received treatment for hypothyroidism. The duration of hypothyroid state is thus clearly distinguished from that of cancer patients, who were athyrotic with cessation of thyroid medication. Furthermore, our study examines both short and long delay retrieval, along with semantic cuing, as compared to a one-time retrieval score without cuing. Specifically, Burmeister et al. $^{2}$ used a 15-item word list that yields a total learning score and a delayed recall score, whereas our study used the CVLT, which yields scores for total learning, short delay recall, long delay recall, and recognition. Though there were no differences for total learning and recognition scores between the hypothyroid participants and comparison subjects, the significant difference between groups for both the short and long delay recall further supports and extends the theory that hypothyroidism is associated with a deficit in synthesis and retrieval of information, rather than the initial formation of memory (encoding).

Though this study is unique in featuring a younger patient group, a large age-matched comparison group, and an extensive neuropsychological battery, it does evidence some limitations. The number of hypothyroid participants is still relatively small and the range of their TSH levels fairly broad. Also, the study includes only subjects with hypothyroidism who stated that they were experiencing subjective cognitive impairment. A comparable study including hypothyroid patients who do not report cognitive problems is also warranted.

This study was supported by Deutsche Forschungsgemeinschaft (grant Ba 1504/3-1) and Thyroid Research Advisory Council (TRAK) (grant SYN-0400-13 to Dr. Bauer).

TABLE 2. Descriptive Statistics and t-Tests for Independent Samples Comparing Healthy Comparison Subjects to Hypothyroid Subjects on All Neuropsychological Tests

\begin{tabular}{|c|c|c|c|c|c|c|}
\hline \multirow[b]{2}{*}{ Test } & \multicolumn{2}{|c|}{$\begin{array}{c}\text { Healthy Comparison } \\
\text { Subjects }(N=10)\end{array}$} & \multicolumn{2}{|c|}{ Hypothyroid Group $(\mathrm{N}=14)$} & \multirow[b]{2}{*}{$\mathbf{t}$} & \multirow[b]{2}{*}{$\mathrm{p}$} \\
\hline & Mean & SD & Mean & SD & & \\
\hline Logical Memory 1 & 28.9 & $(8.41)$ & 26.00 & $(5.46)$ & 1.03 & n.s. \\
\hline Logical Memory 2 & 26.6 & $(7.97)$ & 22.36 & $(7.04)$ & 1.38 & n.s. \\
\hline Trail-Making Test Part A & 30.5 & $(7.76)$ & 29.90 & $(7.01)$ & 0.19 & n.s. \\
\hline Trail-Making Test Part B & 80.9 & (34.02) & 65.50 & (21.19) & 1.37 & n.s. \\
\hline Animal Naming & 22.6 & $(5.08)$ & 19.90 & $(6.40)$ & 1.12 & n.s. \\
\hline FAS & 42.2 & $(9.37)$ & 45.40 & (17.23) & -0.50 & n.s. \\
\hline CVLT Trial 1 & 8.8 & $(1.81)$ & 8.00 & $(2.25)$ & 0.93 & n.s. \\
\hline CVLT Trial 5 & 15.0 & $(1.25)$ & 13.80 & $(2.08)$ & 1.64 & n.s. \\
\hline CVLT Total & 63.2 & $(6.05)$ & 59.60 & $(8.60)$ & 1.14 & n.s. \\
\hline CVLT List B & 8.2 & $(1.32)$ & 7.50 & $(2.38)$ & 0.84 & n.s. \\
\hline CVLT Short Delay Free & 13.2 & $(2.90)$ & 11.40 & (3.18) & 1.39 & n.s. \\
\hline CVLT Short Delay Cued & 14.5 & $(1.78)$ & 12.80 & $(2.12)$ & 2.08 & 0.05 \\
\hline CVLT Long Delay Free & 14.4 & $(1.90)$ & 12.60 & (2.17) & 2.14 & $<0.05$ \\
\hline CVLT Long Delay Cued & 14.9 & (1.66) & 12.70 & $(2.30)$ & 2.56 & 0.01 \\
\hline Rey Copy & 31.1 & (5.43) & 33.36 & $(2.98)$ & -1.31 & n.s. \\
\hline Rey Delay & 18.7 & $(6.32)$ & 18.75 & $(4.97)$ & -0.02 & n.s. \\
\hline
\end{tabular}


VERBAL MEMORY RETRIEVAL DEFICITS AND HYPOTHYROIDISM

\section{References}

1. Whybrow PC, Prange AJ, Treadway CR: Mental changes accompanying thyroid gland dysfunction: a reappraisal using objective psychological measurement. Arch Gen Psychiatry 1969; 20:4863

2. Burmeister LA, Ganguli M, Dodge HH, et al: Hypothyroidism and cognition: preliminary evidence for a specific defect in memory. Thyroid 2001; 11:1177-1185

3. Smith JW, Tudor Evans A, Costall B, et al: Thyroid hormones, brain function, and cognition: a brief review. Neuroscience Biobehav Rev 2002; 26:45-60

4. Whybrow PC, Bauer M: Behavioral and psychiatric aspects of hypothyroidism, in The Thyroid: A Fundamental and Clinical Text, 9th ed. Edited by Braverman LE, Utiger RD. Philadelphia, Lippincott Raven, 2005, pp 842-849

5. Haggerty JJ, Evans DL, Prange AJ: Organic brain syndrome associated with marginal hypothyroidism. Am J Psychiatry 1986; 143:785-786

6. Osterwil D, Syndaulko K, Cohen S, et al: Cognitive function in non-demented older adults with hypothyroidism. J Am Geriatr Soc 1992; 40:325-335

7. Mennemeier M, Garner RD, Heilman KM: Memory, mood, and measurement in hypothyroidism. J Clin Exp Neuropsychol 1993; 15:822-831

8. Dugbartey AT: Neurocognitive aspects of hypothyroidism. Arch Int Med 1998; 158:1413-1418

9. Jaeschke R, Guyatt G, Gerstein H, et al: Does treatment with Lthyroxine influence health status in middle-aged and older adults with subclinical hypothyroidism? J Gen Intern Med 1996; 11:744-749

10. Sheehan DV, Lecrubier Y, Sheehan KH, et al: The Mini-International Neuropsychiatric Interview (MINI): the development and validation of a structured diagnostic psychiatric interview for DSM-IV and ICD-10. J Clin Psychiatry 1998; 59(suppl 20):22-33

11. Wechsler D: Wechsler Memory Scale-Revised Manual. San Antonio, Tex, Psychological Corp, 1987
12. Delis DC, Kramer JH, Kaplan E, et al: California Verbal Learning Test. New York, Psychological Corp, 1983

13. Osterrieth PA: Le test de copie d'une figure complex: contribution à l'étude de la perception et de la memoire. Arch Psychol 1944; 30:286-350

14. Army Individual Test Battery: Manual of Directions and Scoring. Washington, DC, War Department, Adjutant General's Office, 1944

15. Benton AL, Hamsher K deS: Multilingual Aphasia Examination. Iowa City, Iowa, AJA Associates, 1989

16. Goodglass H, Kaplan E: The Assessment of Aphasia and Related Disorders, 2nd ed. Philadelphia, Lea \& Febiger, 1983

17. Perneger TV: What is wrong with Bonferroni adjustments? Br Med J 1998; 136:1236-1238

18. Christensen H, Griffiths K, MacKinnon A, et al: A quantitative review of cognitive deficits in depression and Alzheimer-type dementia. J Int Neuropsychol Soc 1997; 3:631-651

19. Austin MP, Mitchell P, Wilhelm K, et al: Cognitive function in depression: a distinct pattern of frontal impairment in melancholia? Psychol Med 1999; 29:73-85

20. Nebes R, Butters M, Mulsant B, et al: Decreased working memory and processing speed mediate cognitive impairment in geriatric depression. Psychol Med 2000; 30:697-691

21. Comijs HC, Jonker C, Beekman ATF, et al: The association between depressive symptoms and cognitive decline in community-dwelling elderly persons. Int J Geriatr Psychiatr 2001; 16:361-367

22. Nystgrom E, Caidahl K, Fager G, et al: A double-blind crossover 12 month study of L-thyroxine treatment of women with "subclinical" hypothyroidism. Clin Endocrinol 1988; 29:63-76

23. Monzani E, Del-Guerra P, Caraccio N, et al: Neurobehavioral changes in subclinical hypothyroidism. Clin Invest 1993; 71:367371

24. Baldini I, Vita A, Mauri M, et al: Psychopathological and cognitive features of subclinical hypothyroidism. Prog Neuropsychopharmacol Biol Psychiatry 1997; 21:925-935 